\title{
Contribution A La Caracterisation Diagraphique Des Depots Carbonates Du Cretace Du Bassin Sedimentaire De Cote D'ivoire
}

\section{Yao Kouadio Cyrille}

Assistant, Géologue marin, Département de Géoscience Marine, UFR des Sciences de la Terre et des ressources minières, Université Félix Houphouët

Boigny, Abidjan, Côte d'Ivoire

Kouassi Kouamé Alfred

Assistant, Géologue marin, Département des Sciences de la terre, UFR Environnement, Université Jean Lorougnon Guedé, Daloa, Côte d’Ivoire

\section{Boga Atabli Hervé}

Docteur, Géologue marin, Département de Géoscience Marine, UFR des Sciences de la Terre et des ressources minières, Université Félix Houphouët Boigny, Abidjan, Côte d'Ivoire

\section{Monde Sylvain}

Professeur titulaire, Géologue marin, Département de Géoscience Marine, UFR des Sciences de la Terre et des ressources minières, Université Félix

Houphouët Boigny, Abidjan, Côte d'Ivoire

\section{Digbehi Zéli Bruno}

Professeur titulaire, biostratigraphe, Département de Géoscience Marine, UFR des Sciences de la Terre et des ressources minières, Université Félix Houphouët Boigny, Abidjan, Côte d'Ivoire

\section{N'da Loukou Victor}

Docteur, Ingénieur, Géologue, biostratigraphe, spécialiste en stratigraphie séquentielle Société Nationale des Opérations Pétrolières de Côte d'Ivoire(Petroci), Abidjan Côte d'Ivoire

doi: 10.19044/esj.2016.v12n33p391 URL:http://dx.doi.org/10.19044/esj.2016.v12n33p391

\section{Abstract}

To know the petrophysical characteristics associated with sedimentary facies gammay ray logs providing information on the natural radioactivity of sedimentary rocks and the sonic logs which document on facies and petrophysical variations rocks were studied. This study focused on three (3) wells (BH-X, DH-X and MR-X) bored in the offshore basin of Abidjan margin (Côte d'Ivoire) for which the lithogy is constituted of clay alternating with sand an sandstone at the base surmounted by limestone and 
marls which are covered by clay series. It reveals that two deposits phases occurred during the carbonate sedimentation. Through the gamma ray logs, it appears that the clay phases have low radioactivity, which often progress to average values. This radioactivity recorded in clays would be linked to the low organic matter content and leaching reworked elements. In marl phases radioactivity is medium to high indicating a high content of uranium, thorium and potassium deriving from clay minerals. The gamma ray log varies significantly in limestone phases reflecting the heterogeneity of deposits. Strong radioactivity values in some limestone layers are related to their homogeneity and the present of organic matter. Fluctuations result from long-term changes in submarine hydrothermal flow and may indicate significant variations in facies (crystalline limestone, marl and clay). There are more or less dolomitised layers alternating with more homogeneous chemical limestone benches, biogenic limestone debris of organisms and past clay. The sonic log has two phases in the marl and limestone interval. In the marl phase, the acoustic wave velocity is almost constant, but in the limestone phase velocities fluctuate. Indeed, marl interval contains heterogeneous deposits. Generally the velocity is still low in impermeable and little porous clay. In marls, relatively high propagation velocity implies that they are less penetrable as clays because of the presence of fluid. The sonic log fluctuations in the limestone stage reflect the heterogeneity of deposits. Calcareous layers generally have a shorter time of wave propagation, suggesting that they are indurated, recrystallized and homogeneous. By against certain layers are poorly penetrable and show the presence of fluid. The limestone is fractured, so more porous than clay and marl. The porosity may be related to diagenesis after being dessoluted.

Keywords : Diagraphy, gamma ray log, sonic log, Côte d’Ivoire

\section{Résumé}

Pour connaitre les caractères pétrophysiques en rapport avec les faciès sédimentaires, les logs gammay ray fournissant des informations sur la radioactivité naturelle des formations ainsi que les logs sonic qui renseignent sur les variations faciologiques et pétrophysiques des roches ont été étudiés. Cette étude a porté sur trois (3) sondages (BH-X, DH-X et MR-X) effectués dans le bassin immergé de la marge d'Abidjan (Côte d'Ivoire) pour lesquels la lithologie est constituée d'argile à alternances de sable et de grès à la base, surmontée par du calcaire et des marnes qui sont recouvertes par des séries argileuses. Elle révèle que deux phases de dépôts sont intervenues lors de la sédimentation carbonatée. A travers les logs gamma ray, il ressort que les phases argileuses présentent de faibles radioactivités qui évoluent souvent vers des valeurs moyennes. Cette radioactivité enregistrée dans les argiles 
serait lié à la faible teneur en matière organique remaniée et au lessivage des éléments. Dans les phases marneuses la radioactivité est moyenne à élevée indiquant une forte teneur en uranium de thorium et de potassium provenant des minéraux argileux. Le log gamma ray varie significativement dans les phases calcaires traduisant l’hétérogénéité des dépôts. Les fortes valeurs de radioactivité dans certains niveaux calcaires sont liées à leur homogénéité et à la présence de matière organique. Les fluctuations résultent des variations à long terme du flux hydrothermal sous-marin et peuvent indiquer des variations importantes de faciès (calcaire cristallin, marnes et argiles). On trouve des niveaux plus ou moins dolomitisés alternant avec des bancs calcaires chimiques plus homogènes, des calcaires biogéniques à débris d’organismes et des passées argileuses. Le log sonic présente deux phases dans l'intervalle marno-calcaire. Dans la phase marneuse, la vitesse de propagation des ondes acoustiques est quasiment constante, mais dans les phases calcaires les vitesses sont fluctuantes. En effet, l'intervalle marneux renferme des dépôts hétérogènes. Dans l'ensemble la vitesse reste faible dans les argiles imperméables et peu poreuses. Dans les marnes, la vitesse de propagation relativement élevée implique qu'elles sont moins pénétrables que les argiles à cause de la présence de fluide. Les fluctuations du log sonic dans la phase calcaire traduisent l’hétérogénéité des dépôts. Les niveaux calcaires présentent généralement un temps plus court de propagation des ondes, ce qui suggère qu'ils sont indurés, recristallisés et homogènes. Par contre certains niveaux sont faiblement pénétrables et montrent la présence de fluide. Le calcaire est fracturé, donc plus poreux que les argiles et les marnes. La porosité peut être liée à la diagénèse par suite de dissolution.

Mots clés : Diagraphie, log gamma ray, log sonic, Côte d’Ivoire

\section{Introduction}

Le besoin croissant en énergie, et la forte exploitation des réservoirs amenuisent les potentialités des ressources fossiles disponibles. Aujourd'hui d’énormes efforts sont consentis par les sociétés en terme de moyens financiers, de moyens technologiques et au niveau de la recherche pour accroitre les possibilités de découverte de nouveaux gisements. L’outil diagraphique apparait au premier plan de ces méthodes modernes qui ont apporté une amélioration des stratégies dans le secteur pétrolier.

Pour Chapellier et al (2004), les méthodes diagraphiques permettent d’évaluer la qualité des réservoirs, après l'avoir repéré par les méthodes de surface telles que la géologie et la géophysique. Elles peuvent intervenir dans les corrélations par une relation faciès/ diagraphie Hamon (2001), Champetier (1969). 
En Côte d'Ivoire le bassin sédimentaire a fait l'objet de plusieurs programmes de recherche pour en établir une cartographie sédimentologique, et structurale parfaite. Cependant la plupart des travaux effectués aussi bien dans le domaine onshore et dans le domaine offshore notamment ceux de (Yao, 2012 ; Assalé, 2013 ; Kouassi, 2014 ; Yao, 2014...) ; pour ne citer que ceux là ont porté sur la caractérisation sédimentologique et biostratigraphique du bassin sédimentaire ivoirien, mettant ainsi en évidence la stratigraphie du bassin et les environnements de dépôt. Très peu de travaux ont abordé les variations pétrophysiques. Il en ressort que les aspects qui sont liés au caractéristiques pétrophysiques des dépôts du Crétacé méritent d'être traités pour permettre une connaissance plus approfondie des mécanismes sédimentaires et l'évolution des faciès.

Ce présent travail vise une détermination des caractéristiques pétrophysiques des dépôts marno-calcaires du Crétacé du bassin sédimentaire ivoirien. Pour atteindre cet objectif, nous rechercherons les objectifs spécifiques suivants :

- $\quad$ Déterminer la radioactivité naturelle des dépôts marno-calcaires grâce à la diagraphie gamma ray pour comprendre les phases de dépôt ;

- $\quad$ Identifier les caractères pétrophysiques tels que la porosité des dépôts marno-calcaires traversés.

\section{Cadre physique de l'étude}

La Côte d'Ivoire appartient au bouclier ouest l'Africain (Tagini, 1971) qui, avant l'ouverture de l'Atlantique, était en continuité avec celui du Brésil (Blarez, 1986 ; Marinho \& Mascle, 1987).

Selon Pothin et al. (2000), les formations géologiques de la Côte d'Ivoire se répartissent en deux entités chronologiquement distinctes. D'une part un étroit bassin sédimentaire (2,5\%) d'âge secondaire-tertiaire au sud, et d'autre part un socle précambrien qui constitue la majeure partie du territoire ivoirien, soit 97,5\%, (Figure 1).

La marge océanique ivoirienne est une marge passive formée probablement à partir du Jurassique supérieur ou du Crétacé inférieur par l'ouverture d'un rift intracratonique de l'Atlantique équatorial.

Le bassin sédimentaire ivoirien comprend une partie émergée ou "onshore" qui est peu développée $\left(8000 \mathrm{~km}^{2}\right)$ et une partie immergée ou "offshore" plus grande (22000 $\left.\mathrm{km}^{2}\right)$.

Cette étude concerne trois sondages (BN-X, DH-X et MR-X) situés dans le bassin sédimentaire immergé (offshore) sur un transect est-ouest de la marge d'Abidjan (Figure 1). 


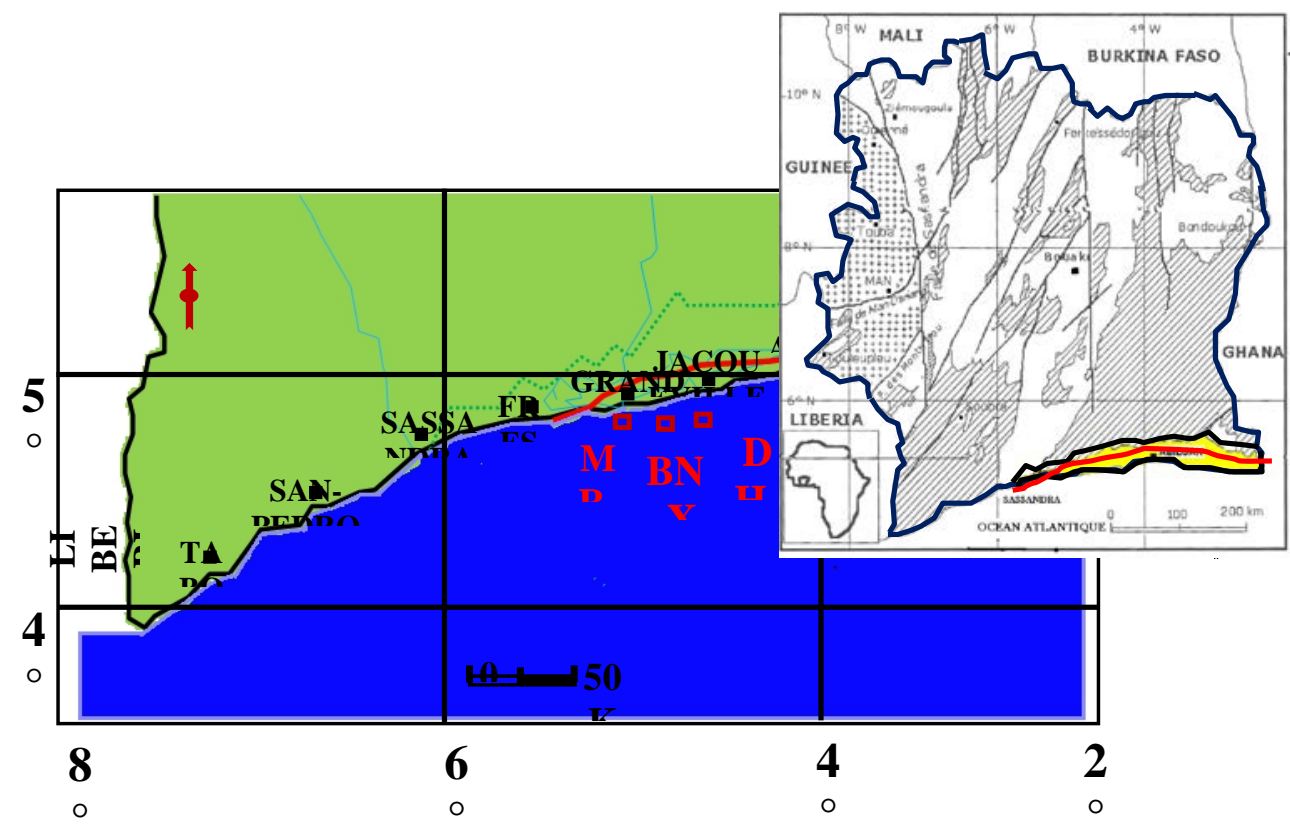

Figure 1:Localisation géographique des sondages étudiés, BN-X, DH-X et MR-X

\section{Légende}
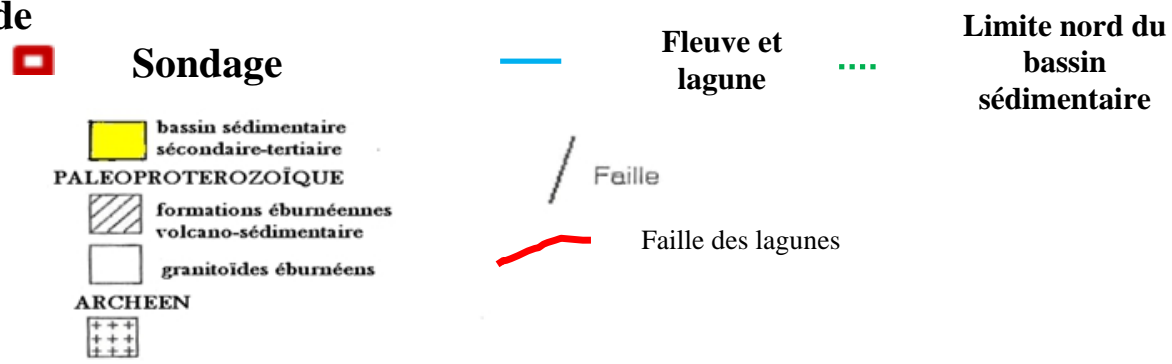

\section{Matériel et méthodes}

La diagraphie est une méthode géophysique permettant d'enregistrer de façon continue des variations de paramètres comme la radioactivité des formations (log gamma ray), la vitesse des ondes acoustiques des terrains traversés (log sonic), la polarisation spontanée (PS), etc., en fonction de la profondeur.

Les données analysées dans ce travail sont des diagraphies différées suivantes :

- Le gamma ray (GR) qui mesure la radioactivité naturelle (Gamma) émise par le Thorium, l'Uranium et le Potassium concentrés dans les formations géologiques ; 
- Le log acoustique ou sonic qui mesure la vitesse des ondes acoustiques à travers les formations. Cette vitesse dépend de la lithologie et de la porosité de la roche traversée.

\section{Etude du log gamma ray}

L’unité de mesure est le microgramme de radium équivalent par tonne, soit 16,5 API (Unité de l'American Petrolium Institut) et la graduation de gauche à droite va de 0 à 10 unités ou de 0 à 150 API.

La lithologie des couches traversées dans un sondage et son contenu en fluide sont déterminés à l'aide de plusieurs techniques d’interprétation. La plus courante consiste à superposer certains enregistrements à partir d'une échelle convenable.

Les sables et les argiles peuvent être déterminés par la courbe gamma-ray (Fig. 2). Dans les sables, le gamma-ray est faible et évolue à gauche de la ligne de radioactivité des argiles. Dans les couches argileuses le rayonnement gamma est élevé et la courbe se situe à droite de la ligne de radioactivité des argiles. Le log gamma ray permet ainsi de distinguer les faciès sédimentologiques.

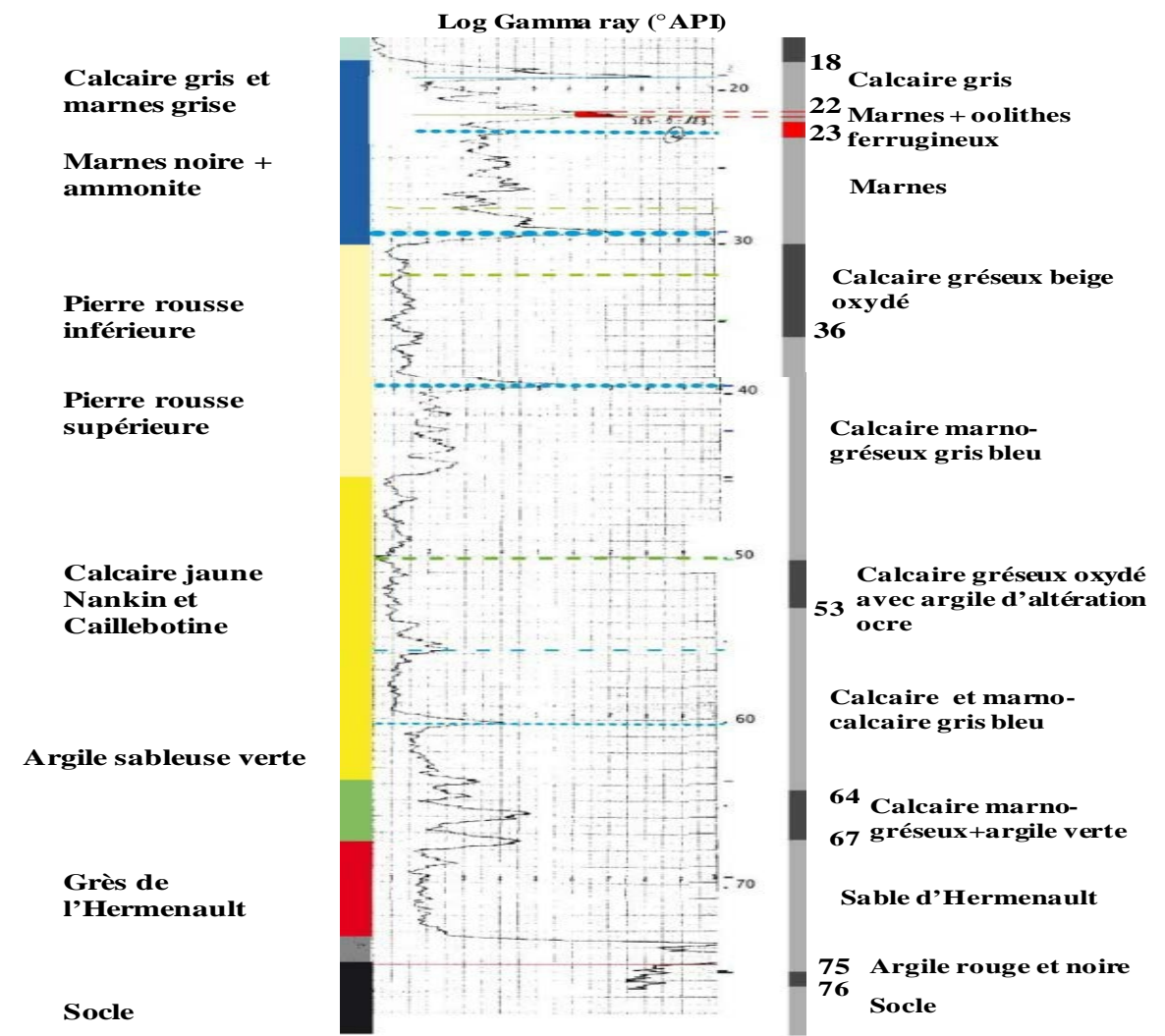

Figure 2 : Analyse lithostratigraphique et comparaison avec le log gamma ray (Chadelle, 2012). 
Le tableau I constitue un modèle d'interprétation sédimentologique des terrains carbonatés en fonction des données spectrales du gamma ray (Glover 2010)

Tableau I: Interprétation sédimentologique des données spectrales du gamma-ray (Glover, 2010).

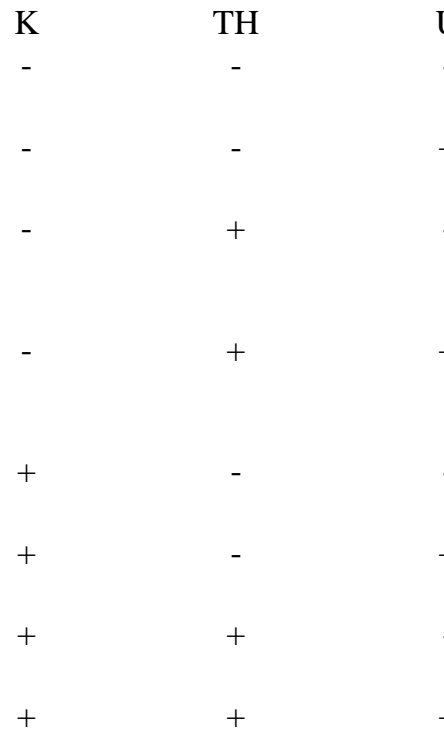

$\mathrm{U}$

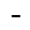

$+$

\section{EXPLICATIONS}

Carbonate pur, absence de matière organique ou environnement oxydant

Carbonate pur, présence de matière organique, environnement réducteur

Pas de carbonate ou carbonate argileux avec des minéraux argileux à rare K et Th élevé. Absence de matière organique, environnement oxydant

Pas de carbonate ou carbonate argileux avec des minéraux argileux à rare $\mathrm{K}$ et Th élevé, présence de matière organique, environnement réducteur

Carbonate glauconieux, absence de matière organique ou environnement oxydant

Carbonate d'origine algaire ou présence de glauconie, de matière organique, environnement réducteur

Carbonate argileux, absence de matière organique ou environnement oxydant

Carbonate argileux, présence de matière organique ou environnement réducteur

+ : Présence ; - : Absence

Ainsi la radioactivité des formations qui est liée à leur composition chimique permet-elle de déterminer les caractéristiques chimiques et faciologiques et d'en déduire les paléo-environnements.

\section{Etude du log sonic}

On regroupe sous le nom de diagraphies acoustiques, les diagraphies qui enregistrent un paramètre en liaison avec l'envoi d'un train d'ondes dans la formation.

Les logs acoustiques ont pour buts de déterminer la porosité des roches réservoirs, la résistivité de l'eau des gisements et le type de fluide présent dans le réservoir. Les couches argileuses ont une vitesse sonique faible. La figure 3 donne la signature des ondes sonics dans un réservoir carbonaté (Nacer Bey \& Chibah, 2010).

L'analyse du log diagraphique se fait en découpant les intervalles en unités dans lesquelles le temps moyen de pénétration des ondes est déterminé. Sur l'enregistrement le temps de parcourt diminue de gauche à droite (de 140 à $40 \mathrm{~ms}$ par pieds) et la vitesse croît inversement. Cette analyse permet de faire une interprétation diagénétique, faciologique et paléo-environnementale. 


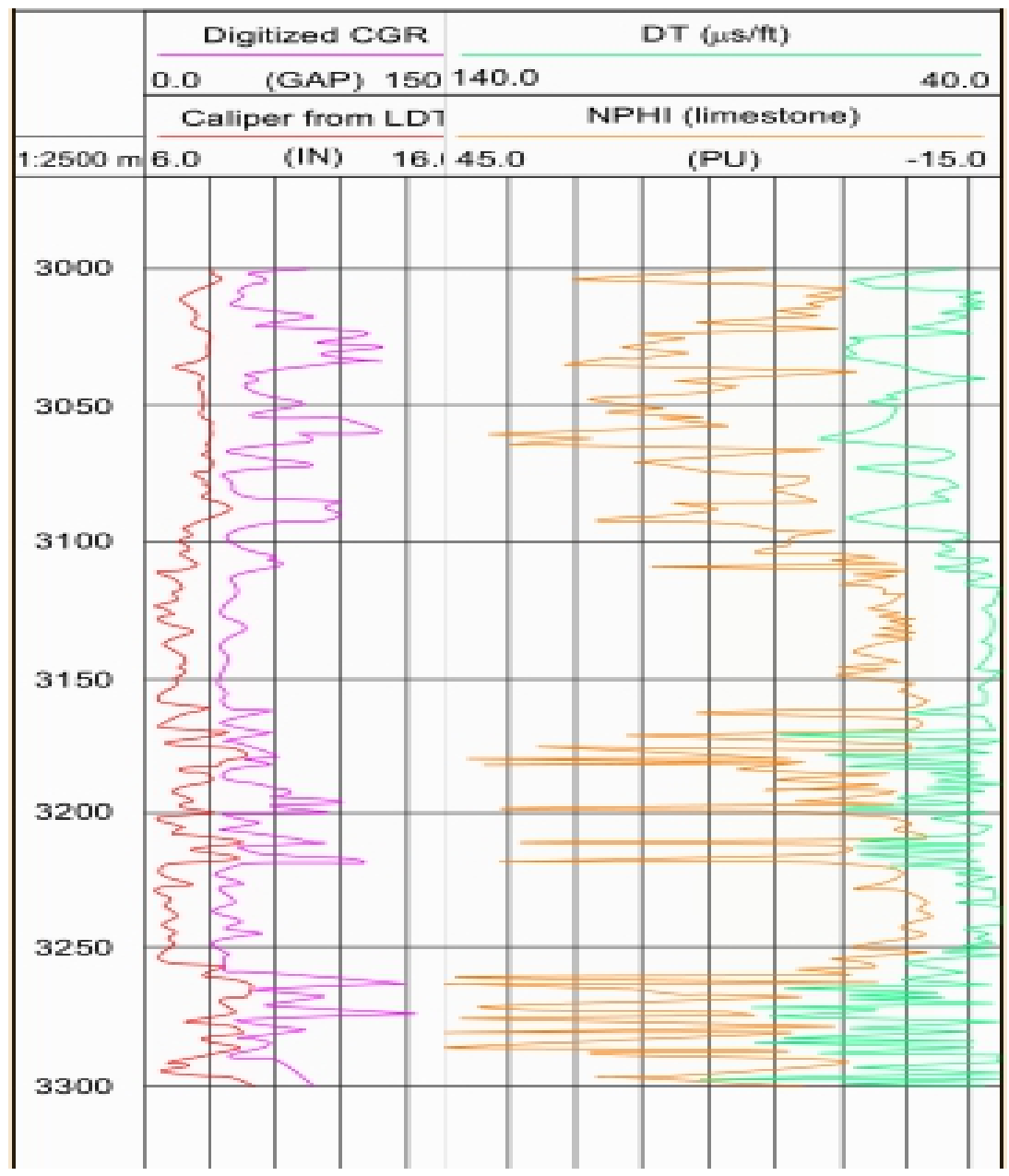

Figure 3 : Log diagraphique composite (gamma-ray, résistivité, sonic et porosité neutron) dans un réservoir carbonaté d’Algérie (Nacer Bey \& Chibah, 2010).

\section{Méthode d'analyse des logs diagraphiques}

\section{$>\quad$ Le log gamma ray}

Les valeurs de radioactivité inférieures ou égales à 40 API sont dites faibles. Celles qui sont comprises entre 40 et 80 API sont moyennes et celles qui sont supérieures à 80 API sont fortes.

Le log sonic

Le temps de propagation des ondes acoustiques est court quand il se situe entre 0 et $60 \mu \mathrm{s}$. il est moyen entre 60 et $100 \mu$ s et élevé lorsqu'il est supérieur à $100 \mu \mathrm{s}$ 


\section{Résultats et interprétations}

\section{Diagraphies gamma ray et sonic du sondage $\mathrm{BN}-\mathrm{X}$}

La figure 4 présente les logs gamma ray et sonic des intervalles carbonatés du sondage $\mathrm{BN}-\mathrm{X}$.

La lithologie est formée d'argiles gris sombre indurées massive intercalées de bancs de grès à grains fins à la base. Cette série est surmontée par du calcaires gris clair beige et indurés avec des intercalations de marnes gris clair, indurée, fissile à subfissile et des argiles calcaires gris sombre. Le sommet est essentiellement argileux.

\section{Analyse}

\section{$>\quad$ Le log gamma ray}

Il montre deux grandes phases dans les dépôts carbonatés :

- $\quad$ Première phase (2408 m - 2548 m)

Dans les niveaux argileux, les valeurs du gamma ray sont globalement faibles (53 API) avec une moyenne de 40 API, alors qu'elles sont plus élevées (73 API) dans les niveaux marneux où la moyenne est de 55 API.

- $\quad$ Deuxième phase (2548 m-2621 m)

L'intervalle constitué de calcaire à passées d'argiles et de marnes se caractérise par l'oscillation des valeurs du gamma ray. Dans le calcaire les valeurs sont relativement faibles (30 API à 35 API) avec une moyenne de 32 API. Deux niveaux calcaires situés à $2590 \mathrm{~m}$ et $2596 \mathrm{~m}$ présentent des valeurs plus élevées (75 API). Dans les marnes les valeurs du gamma ray sont faibles à moyennes (40 API à 60 API). Les valeurs dans les argiles sont moyennes (60 API).

$>\quad$ Le $\log$ sonic

Le log sonic présente également deux phases dans les intervalles carbonatés :

- $\quad$ Première phase (2408 m- 2548 m)

Cet intervalle est constitué d'argiles à passées de marnes. Son log sonic présente les caractéristiques suivantes :

- Dans les niveaux marneux, le temps de propagation des ondes

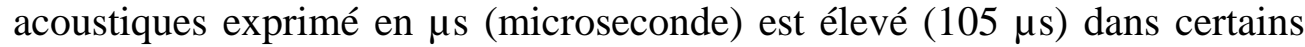
niveaux (2408 met $2471 \mathrm{~m}$ ) et se traduit par une faible vitesse de propagation. Dans d'autres niveaux marneux (2423 m et $2459 \mathrm{~m}$ ) ce temps reste faible (88 $\mu \mathrm{s})$, ce qui implique une vitesse de propagation élevée.

- $\quad$ Dans les niveaux argileux, le log sonic est très fluctuant avec une valeur moyenne de $98 \mu$ s correspondant à une vitesse de propagation moyenne.

- $\quad$ Deuxième phase (2548 m-2621 m) 
Dans l'intervalle le log sonic est très fluctuant. Sa valeur est élevée dans le niveau calcaire $2548 \mathrm{~m}$ (114 $\mu$ s) et faible dans l'intervalle $2575 \mathrm{~m}$ à

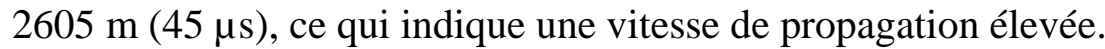

Dans les marnes, le temps de propagation élevé (93 $\mu$ s) traduit une vitesse de propagation faible. Dans les argiles la vitesse est élevée par rapport au temps de propagation (65 $\mu$ s) des ondes acoustiques.

\section{Interprétation}

\section{$>\quad$ Log gamma ray}

Le log gamma ray varie significativement dans les niveaux calcaires mais présente peu de variation dans les niveaux marneux.

- $\quad$ Première phase (2408 m- 2548 m)

La faible valeur du gamma ray dans les argiles de l'intervalle indique un lessivage des sédiments (Schlumberger, 1989). La radioactivité élevée dans les marnes indique une forte teneur en uranium, en thorium et en potassium.

- $\quad$ Deuxième phase (2548 m-2621 m)

Les fluctuations de la radioactivité traduisent l'hétérogénéité des dépôts. Les fortes valeurs de radioactivité dans certains niveaux calcaires suggèrent une évolution géochimique des éléments comme le l'uranium qui se concentre dans le calcaire pur riche en matière organique.

Les fluctuations enregistrées résultent des variations à long terme du flux hydrothermal sous-marin. Les évolutions à court terme sont dues aux variations du niveau marin avec l'alternance des transgressions et régressions qui provoquent des variations des sorties d'éléments dans le plateau continental. D'autres phénomènes comme le bilan érosif du continent ou les conditions d'oxygénation de l'océan (importance des périodes anoxiques) peuvent s'ajouter ou contrarier les effets des causes principales. 


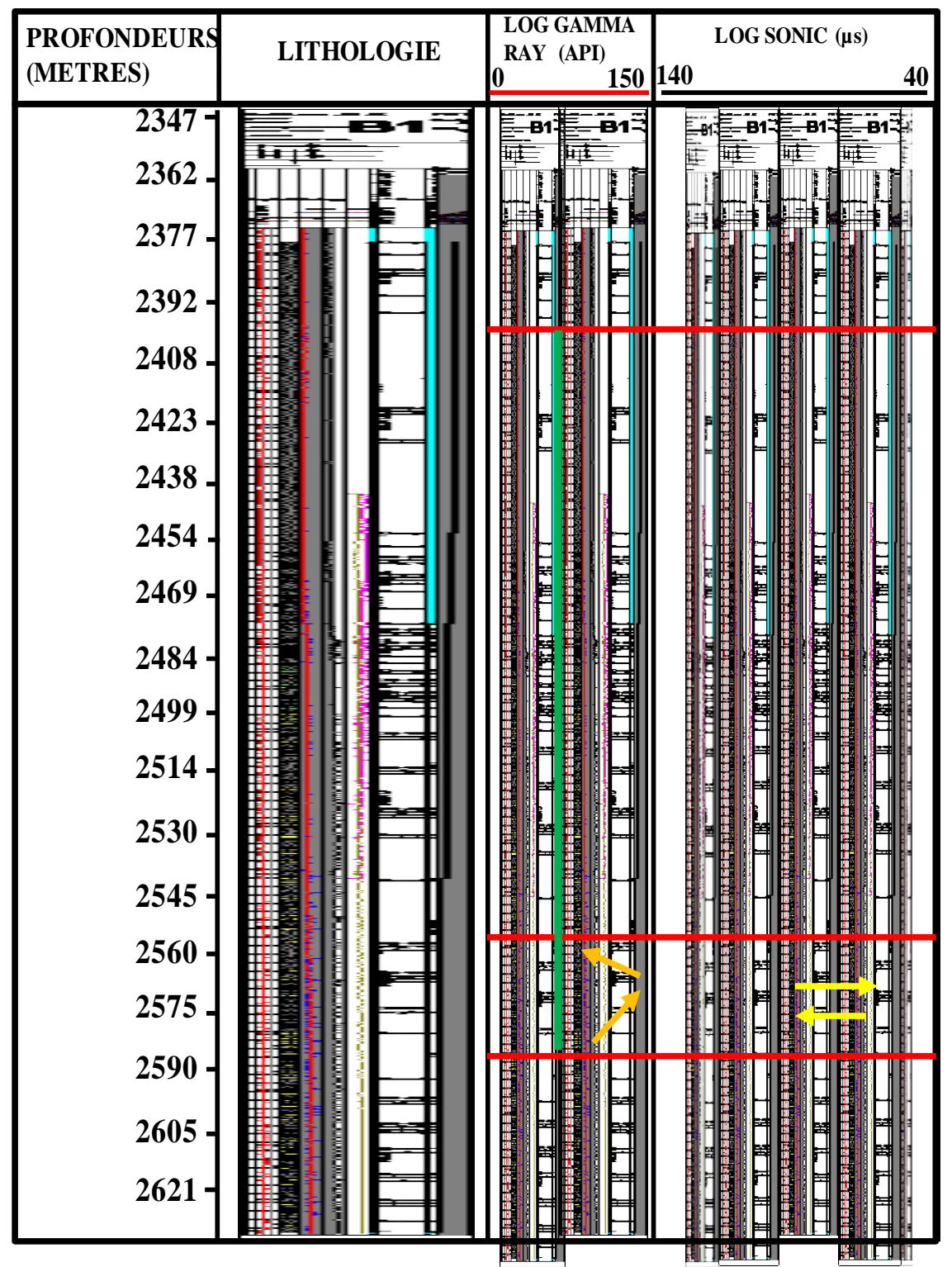

Figure 4 : Logs diagraphique gamma ray et sonic des intervalles carbonatés du sondage $\mathrm{BN}-\mathrm{X}$.

Ligne de la moyenne des argiles

\section{$>\quad$ Log sonic}

Le log sonic varie également dans les niveaux calcaires mais reste pratiquement constant dans les niveaux marneux.

- $\quad$ Première phase (2408 m- 2548 m)

Le temps de propagation des ondes est globalement moyen en dépit de quelques variations. Il indique que l'intervalle renferme des dépôts 
hétérogènes. Dans l'ensemble la vitesse reste faible dans les argiles imperméables et peu poreuses. Dans les marnes, la vitesse de propagation moyenne est plus élevée, ce qui implique qu'elles sont moins pénétrables que les argiles à cause de la présence de fluide. Ces niveaux sont donc relativement poreux (porosité matricielle).

- $\quad$ Deuxième phase (2548 m-2621 m)

Les fluctuations du log sonic traduisent l’hétérogénéité des dépôts. Les niveaux calcaires présentent généralement un temps plus court de propagation des ondes, ce qui suggère qu'ils sont indurés, recristallisés et homogènes. Par contre certains niveaux sont faiblement pénétrables et montrent la présence de fluide. Le calcaire est fracturé, donc plus poreux que les argiles et les marnes. La porosité peut être liée à la diagénèse par suite de dissolution.

\section{Diagraphies gamma ray et sonic du sondage $\mathrm{DH}-\mathrm{X}$}

La figure 5 présente les logs diagraphiques gamma ray et sonic des intervalles carbonatés du sondage DH-X.

La sédimentation est composée d'une alternance d'argiles gris brunâtre à gris sombre friable souvent très micacée et silteuse avec des sables translucides à grains fins à moyens et présente une intercalation de grès à la base. Cette série de base est surmontée par une alternance de marnes et de calcaire à intercalation d'argile. La série terminale est constituée d'argiles gris sombre, indurées, faiblement silteuse et glauconieuse.

\section{Analyse}

\section{$>\quad$ Le log gamma ray}

Ce log présente deux grandes phases de dépôts carbonatés :

- $\quad$ Première phase (2225-2377 m)

Les niveaux marneux ont des pics moyens de 100 API alors que d'autres présentent des signatures de moyenne amplitude, soit 80 API en moyenne. Dans l'intervalle, les argiles enregistrent de valeurs moyennes de radioactivité (moyenne de 70 API). Par ailleurs, on trouve deux niveaux marneux (2289-2313 m) à très fortes valeurs (130 API).

- $\quad$ Deuxième phase (2377-2453 m)

Elle est caractérisée par une fluctuation importante du log gamma ray. Dans les calcaires les valeurs sont voisines de 150 API et sont moyenne dans les marnes (80 API). Les niveaux argileux présentent des valeurs élevées mais plus faibles que celles enregistrées dans les niveaux calcaires (100 API)

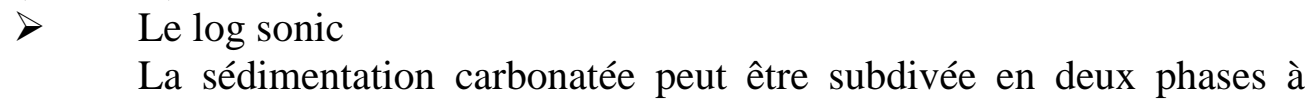
partir du log sonic : 
- $\quad$ Première phase (2225-2377 m)

L'évolution du log sonic de l'intervalle présente deux phases. La première au sommet (2225 et $2333 \mathrm{~m}$ ) est caractérisée par un temps de propagation élevé (moyenne de $123 \mu$ s). La deuxième à la base (2333 à 2377 m) se caractérise par un temps de propagation plus faible (moyenne $106 \mu \mathrm{s}$ ). On note cependant que les temps de propagation sont plus courts dans les niveaux marneux, notamment à 2353 et $2371 \mathrm{~m}$ (90 $\mu \mathrm{s})$.

- $\quad$ Deuxième phase (2377-2453 m)

Il se caractérise également par une forte oscillation du log sonic. Les calcaires ont un faible temps de propagation (moyenne $66 \mu \mathrm{s}$ ), impliquant une vitesse de propagation élevée du son. Les niveaux argileux ont des valeurs moyennes de $90 \mu \mathrm{s}$, ce qui implique une vitesse de propagation moyenne. Certains niveaux argileux, notamment à $2423 \mathrm{~m}$ ont un temps plus long (123 $\mu \mathrm{s})$. Les passées marneux ont des valeurs moyennes de propagation du son (86 $\mu$ s).

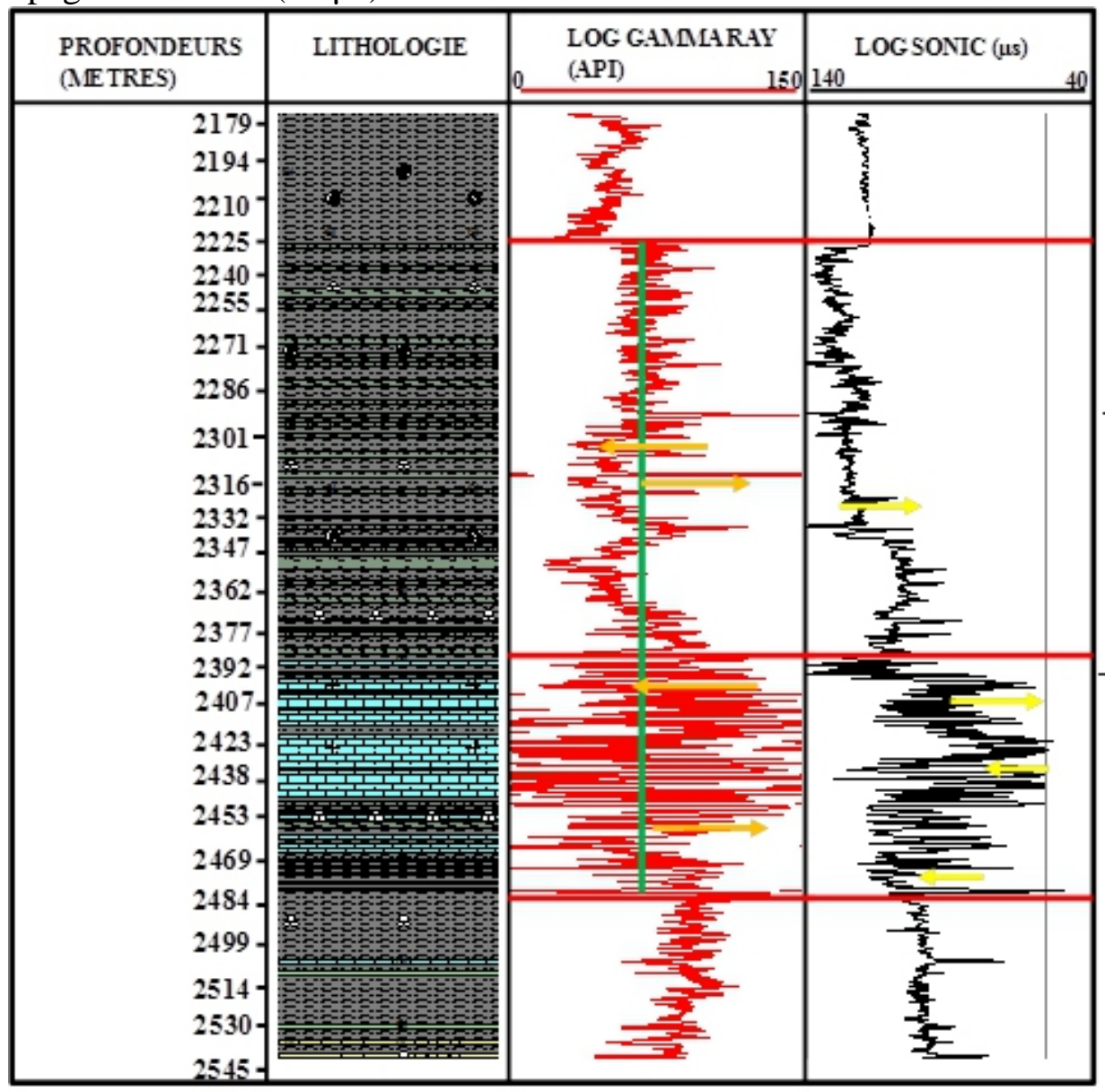

Figure 5: Logs diagraphiques gamma ray et sonic des intervalles carbonatés du sondage DH-X. 


\section{Interprétation}

$>\quad$ Le log gamma ray

Le log gamma ray présente de fortes variations dans les niveaux calcaires et varie modérément dans les niveaux marneux.

- $\quad$ Première phase (2225-2377 m)

La radioactivité des marnes est nettement plus forte que dans les argiles où la signature du gamma ray est similaire à celle des sables. Cela pose un problème d'interprétation. On pourrait expliquer les valeurs fortes du gamma ray des marnes à une plus forte concentration d'uranium, de thorium et de potassium provenant des minéraux argileux.

- $\quad$ Deuxième phase (2377-2453 m)

La forte radioactivité des calcaires est liée à leur homogénéité et à la présence de matière organique. Les fluctuations des valeurs dans l'intervalle indiquent des variations importantes de faciès (calcaire cristallin, marnes et argiles). L'intervalle est dominé par des calcaires avec des faibles variations lithologiques. On trouve des niveaux plus ou moins dolomitisés alternant avec des bancs calcaires chimiques plus homogènes, des calcaires biogéniques à débris d'organismes et des passées argileuses.

$>\quad$ Le log sonic

Le log sonic présente dans les niveaux calcaires des changements importants mais reste à peu près homogène dans les niveaux marneux.

- $\quad$ Première phase (2225-2377 m)

Au sommet de l'intervalle les argiles et les passées marneuses sont moins indurées et plus difficiles à pénétrer par les ondes. La base plus indurée et riche en carbonate montre des vitesses de propagation plus élevées. Certains niveaux marneux plus indurés du fait d'une recristallisation importante enregistrent une vitesse plus rapide que dans les aux autres niveaux.

- $\quad$ Deuxième phase (2377-2453 m)

Le calcaire plus homogène et consolidé par recristallisation enregistre des vitesses élevées. Les argiles et les marnes moins homogènes et moins consolidées ont des vitesses de propagation faibles à moyennes.

\section{Diagraphies gamma ray et sonic du sondage MR-X}

La figure 6 donne les logs diagraphiques gamma ray et sonic des intervalles carbonatés du sondage MR-X.

La lithologie est constituée d'argiles gris sombre, indurées à friables, massives et légèrement silteuses à intercalations de grès indurés à friable à la base. Ces dépôts sont surmontées par du calcaire brun clair, induré, cristallin et faiblement argileux et des intercalations d'argiles gris sombres indurées et massives. Le sommet est une série argileuse à intercalations de calcaire et de grès. 


\section{Analyse}

Le log gamma ray

Deux phases de dépôts sont mises en évidence dans la sédimentation carbonatée :

- $\quad$ Première phase (2700- 2900 m)

$>\quad$ Le log sonic

L’évolution du log sonic permet de distinguer deux phases.

- $\quad$ Première phase (2700-2900 m)

Le temps de propagation est moyen (100 $\mu$ s) à la base (2860-2900 $\mathrm{m})$, court $(87 \mu \mathrm{s})$ au milieu $(2750-2860 \mathrm{~m})$ et plus élevé (107 $\mu \mathrm{s})$ au sommet (2700-2750 m)

\begin{tabular}{lrlrl} 
& \multicolumn{2}{l}{ LOG GAMMA } & \multicolumn{2}{c}{ LOG SONIC $(\mu \mathrm{s})$} \\
$\begin{array}{l}\text { PROFONDEURSLITHOLOGIE } \\
\text { (METRES) }\end{array}$ & $\begin{array}{l}\text { RAY(API) } \\
0\end{array}$ & 150140 & 40
\end{tabular}
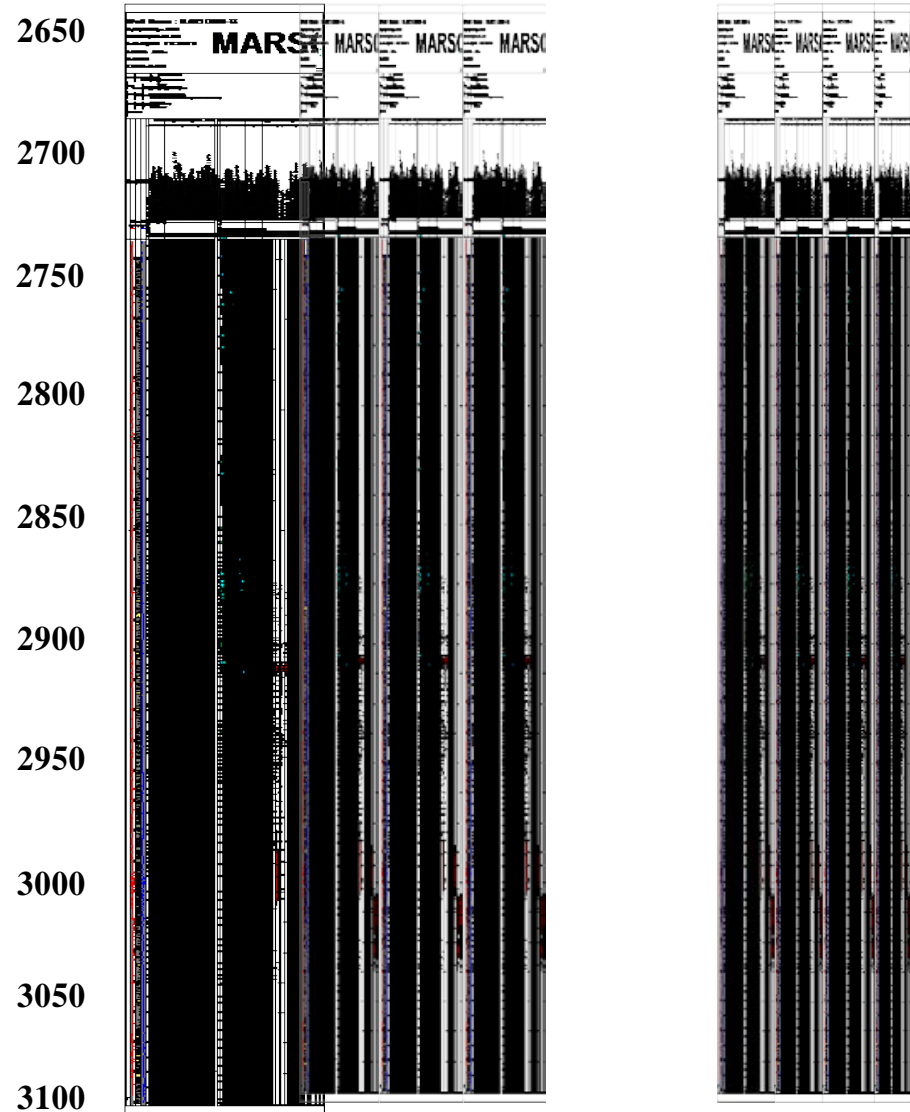

Figure 6: Logs diagraphiques gamma ray et sonic des intervalles carbonatés du sondage MR-X. 


\section{Interprétation}

$>\quad$ Le log gamma ray

Les variations constatées ont des implications diverses au plan pétrophysique dans les phases carbonatées :

- $\quad$ Première phase (2700- $2900 \mathrm{~m}$ )

Les plus faibles valeurs du gamma ray des argiles par rapport aux marnes indiquent que les argiles ont une faible concentration en minéraux radioactifs. Cela peut s'expliquer par la faible teneur en matière organique remaniée et au lessivage des éléments.

- $\quad$ Deuxième phase (2900-3000 m)

La radioactivité de l'intervalle est en relation avec la concentration en carbonates. Cela signifie que l'accumulation d'éléments radioactifs est en relation avec les dépôts fins. L'accumulation de matière organique et la présence de constituants minéraux favorisent la concentration en éléments radioactifs. La concentration des constituants minéraux favorise la dilution des éléments radioactifs. La présence d'uranium associée à une déflexion du gamma ray indiquerait la présence de stylolithes et de fractures ou traduirait la qualité des calcaires car le gamma ray élevé correspond à un calcaire de bonne qualité et à l'augmentation de la dolomitisation (Ballay \& Roy, 2005).

Le log sonic

Les vitesses des ondes acoustiques enregistrées dans les intervalles carbonatés suggèrent une variation des caractères pétrophysiques des roches.

- $\quad$ Première phase (2700- 2900 m)

L'intervalle comprend trois niveaux. Le premier niveau marneux (2700-2750 m), plus hétérogène et moins induré a une vitesse de propagation plus faible. Le deuxième (2750-2860 m) plus homogène et induré a une vitesse de propagation plus élevée. La base (2860-2900 m) comprend des dépôts mixtes moyennement indurés et enregistre des vitesses moyennes de propagation du son.

- $\quad$ Deuxième phase (2900-3000 m)

Le temps de propagation faible à moyen caractérise des dépôts hétérogènes. Cette zone à dépôts mixtes comprend des calcaires peu indurés et hétérogènes.

\section{Discussion}

Le log gamma ray des différents sondages montre des fluctuations concentriques dans le calcaire. Les valeurs des rayonnements gamma des calcaires sont généralement supérieures à celles enregistrées dans les argiles. Dans les marnes, les pics isolés permettent de les identifier dans les couches argileuses. 
D’après Beghoul (1991), l'enrichissement momentané en élément radioactif peut produire une granodécroissance. Les mécanismes hydrologiques locaux, les mouvements eustatiques, la modification progressive des sources de matériel et des conditions de sédimentation influent sur la concentration d'éléments radioactifs au cours de la transgression. Ces mécanismes induisent des variations de concentration d'éléments radioactifs. Ces résultats s'accordent avec ceux du présent travail.

Champetier (1969) indique pour sa part que la radioactivité des calcaires est faible et ne se distingue pas de celle des autres formations. Les argiles et les marnes ont souvent une radioactivité beaucoup plus forte que celle des autres roches. Il montre que les éléments radioactifs se déposent comme constituants de certains minéraux (sable feldspathique, minéraux lourds) et inclus dans les sédiments, ou précipités par voie physicochimique. Il indique que la migration ou le dépôt de l'uranium et du thorium pendant la sédimentation est intimement liée aux processus d’oxydo-réduction. La précipitation d'uranium en milieu marin peut résulter d'une réduction de l'état hexavalent à l'état quadrivalent par l'action du sulfure d'hydrogène dans les grands fonds. Cela peut expliquer les fortes radioactivités observées dans les marnes et les argiles marines qui caractérisent un milieu réducteur. Il explique aussi que dans les calcaires ou calcaires marneux on peut avoir une augmentation de la radioactivité et de la teneur en argiles.

Pour Hissein (2011) la diminution des valeurs dans la courbe gamma ray s'explique par la présence de calcite, minéral dépourvu d'éléments radioactifs $\left(\mathrm{CaCO}_{3}\right)$. L'auteur a montré qu'une fluctuation du gamma ray traduit l'augmentation progressive de calcaire et d'alternances calcaireargile. Ses travaux montrent qu'une diminution du gamma ray dans un calcaire caractérise un calcaire propre.

Schlumberger (1989) a montré que la faible radioactivité des séries argileuses traduit un lessivage des éléments. Cela explique la faible radioactivité des argiles des sondages étudiés. D’après Matt (2012), la forte radioactivité des sédiments traduit une forte concentration d'uranium provoquée par la matière organique et le dépôt des black shales.

La diagraphie sonic se caractérise par des fluctuations concentriques dans le calcaire et la vitesse des ondes acoustiques y est plus forte que celle des argiles. On distingue des pics de vitesse pour certains niveaux marneux, mais la vitesse reste faible dans l'ensemble. Carrara (2010) et nous même dans ce mémoire avons montré que l’hétérogénéité des roches provoque des variations continues le long des diagraphies sonic car l'énergie acoustique réfléchie dépend du milieu percuté par l'onde. Les couches continues ont se caractérisent par une vitesse de propagation des ondes acoustiques supérieure aux couches discontinues. Ehrenberg et al (2002) ont montré que la porosité des roches est liée à la diagenèse et à la dissolution. Leurs travaux montrent 
que toute région affectée par des transgressions et régressions est affectée par des variations latérale et verticale de faciès. Ces phénomènes peuvent modifier sensiblement les caractéristiques pétrophysiques des roches et induire une variation de leur signature diagraphique.

\section{Conclusion}

Les sondages étudiés sont constitués à la base d'argile avec des alternances de sable et de grès surmontée de calcaire et de marnes et se terminent par des séries argileuses.

Les intervalles carbonatés sont calcaires à la base et marneux au sommet. Les calcaires présentent dans l'ensemble une fluctuation du log gamma ray suggérant un calcaire non propre. Le rayonnement gamma donne des valeurs souvent supérieures à celles des argiles. Cela est lié à la forte teneur en argiles et à la décomposition de la matière organique. Par ailleurs les argiles subissent un lessivage et libèrent des éléments comme l'uranium qui s’accumule dans les calcaires plus fins. Les marnes présentent parfois des valeurs supérieures à celles des argiles en raison de la diagénèse de la matière organique. Les minéraux argileux sont souvent lessivés et donnent de faibles rayonnements, provoquant la faible radioactivité des marnes qui les renferment. Les calcaires enregistrent des vitesses de propagation sonique élevées. Cela montre que ces dépôts sont indurés et homogènes et que la diagenèse s'est faite de façon presque constante. La recristallisation est prédominante lors de la diagénèse tardive. Dans les marnes, les ondes sonores se propagent lentement dans l'ensemble, ce qui caractérise des dépôts hétérogènes peu indurés. Des variations latérales et verticales s’observent dans l'ensemble de l'intervalle carbonaté. Elles indiquent la modification des caractéristiques pétrophysiques des roches en rapport avec la diagénèse (dissolution) et traduisent les fluctuations eustatiques.

\section{References:}

1. Assalé F.Y. P (2013)- Caractérisation sédimentologique, palynologique, géochimique et paléoenvironnementale des formations sédimentaires connexes à la faille des lagunes (Est du bassin onshore de Côte d'Ivoire). Thèse de doctorat, Univ F.H.B Cocody 361p.

2. Ballay R. E \& Roy R. E (2005) - Formation evaluation: Carbonate versus sandstone. Robert E Ballay, LLC. 20 p.

3. Beghoul M. S. (1991) - Apport et contribution de l'analyse de diagraphies à la connaissance d'un bassin sédimentaire. Application au bassin de Timimoun (Algérie). Thèse Université Louis Pasteur de Strasbourg : $266 \mathrm{p}$. 
4. Blarez E., (1986)- La marge continentale de Côte d'Ivoire Ghana, marge continentale transformante. Thèse Doctorat de l'Université de Paris VI : $188 \mathrm{p}$.

5. Carrara A. (2010) - Etude de la faisabilité de l’analyse et de la variabilité spatiales des résistances au cisaillement de la fondation et de l'interface roc/béton du barrage de la Turquie et de rapide « 7 ». Mémoire Diplôme d’Ingénieure Option Génie Civil : 135 p.

6. Chadelle B. (2012) - Méthodes d’évaluation des logs géologiques (Bassin Aquitaine-Jurassique inférieur et moyen) à partir de diagraphie Gamma-Ray et afin de quantifier la validité des données de la BSS. Mémoire de Master, Université de Bordeaux : 62 p.

7. Champetier D. R. (1969) - Application de la diagraphie de radioactivité naturelle à l'étude de deux déblais de l'autoroute A6. Laboratoire Central des Ponts et Chaussée et Laboratoire Régional d’Autun. «Bulletin de Liaison du Labooratoire Routier des Ponts et Chaussées», 39: 168 p.

8. Chapellier D., Mari J. - L., Tacchini G., Baron L., Monnet R., Marescot L., Rochat S., Furrer J., Perret F. (2004) - Cours de Diagraphies différées: option Réservoir. Cours online de géophysique. http:// www.ig.unil.ch/cours/, Institute de Géophysique (Université de Lausanne). Institut française de Pétrole (IFP). 78 p

9. Ehrenberg S. N., Pickard A. H., Svana T. A \& Oxtoby N. H (2002)Cement geochemistry of photozoan carbonate strata (upper carboniferous-lower Permian), Finmark carbonate platform, Barents Sea. «Journal of Sedimentary Research», 72: pp.95-115.

10. Hamon Y (2001)- Sédimentologie, géométrie et signification géodynamique des dépôts réservoirs silicoclastiques du Trias/ Rhétien du Chemery (Sologne, SW du bassin de Paris). Diplôme d'Etudes Approfondies (Structure et Evolution de la Lithosphère). Faculté des Sciences et Techniques du Languedoc, Montpellier II : 40 p.

11. Hissein D. A. (2011)- Basses terres du Saint-Laurent et leur potentiel en hydrocarbures. Mémoire de Maîtrise en Science de la Terre de l'Université de Laval Quebec : 158 p.

12. Glover P (2010)- Exploitation de gaz de shale dans les basses terres du Saint-Laurent: Aspect technique et de sécurité, présentation, Univ. Laval, Quebec, Canada. Ground Water Protection Council (2009), State Oil and Natural Gaz regulations, Designed to Protect Water Resources: 65 p.

13. Kouassi K. A. (2014)- Episode anoxique à la limite Cénomanien/Turonien dans le basin sédimentaire de Côte d’Ivoire : intérêt pétrolier des black shales. Doctoract Thèse unique, Univ Félix 
Houphouët Boigny (Abidjan/Cocody) : 275 p.

14. Matt H. (2012) - Interpreting spectral gamma-ray logs [blog Post]. De http://agilegeoscience.com/journal/2013/2/26/interpreting-spectralgamma-ray-logs.html

15. Nacer Bey R. \& Chebah R. (1994) - Réservoir carbonatée en Algérie. Contribution de Sonatrach, Division Exploration: 15 p.

16. Pothin K. B., Gion P. \& Groayes C. C. (2000)- Bilan géochronologique du socle précambrien de Côte d’Ivoire. Bioterre « Revue Internationale des Sciences de la Vie et la Terre », 1 (1): 3647.

17. Schlumberger (1989)- Log interpretation. Principles/Applications. Schlumberger Educational Service, Houston (Texas): 223 p.

18. Tagini B. (1971)- Esquisse structurale de la Côte d'Ivoire. Essai de géotectonique régionale. Thèse doctorat de l'Université de Lausanne et Bulletin de la Société de Développement Minière de Côte d'Ivoire (SODEMI), Abidjan, 5: $302 \mathrm{p}$.

19. Yao K. C. (2014)- Caractérisation des niveaux carbonatés du Crétacé du bassin sédimentaire de Côte d'Ivoire : intérêt pétrolier et implication paléoenvironnementale. Thèse de Doctorat, Univ F.H.B Cocody: $228 \mathrm{p}$.

20. Yao N. J-P (2012)- Caractérisation Sédimentologique, minéralogique, géochimique et biostratigraphique des falaises vives de Fresco: région de Grand-Lahou (Côte d'Ivoire). Thèse de Doctorat, Univ F.H.B Cocody: 187 p. 